\title{
Circular RNAs in head and neck cancer diagnosis and potential molecular targeting
}

\author{
Fatemeh Momen-Heravi ${ }^{*}$ and Shashi Bala ${ }^{2}$ \\ ${ }^{1}$ Division of Periodontics, Section of Oral and Diagnostic Sciences, Columbia University College of Dental Medicine, USA \\ ${ }^{2}$ Department of Medicine, University of Massachusetts Medical School, USA
}

\begin{abstract}
Circular RNAs (circRNAs) are a class of non-coding RNAs which is being explored recently. CircRNAs are highly conserved across species and exhibit tissue/ developmental stage-specific expression. circRNAs are more stable than linear mRNAs and lack a 5'-terminal cap and 3'-terminal poly A tail. CircRNAs modulate gene expression at the transcriptional and post-transcriptional levels. circRNAs play different functional roles in hallmark cancer events both as oncogens and tumor supressors including regulation of angiogenesis, induction of metastasis and invasion, evading cell death. Here, we outlined the molecular mechanisms of circRNA function to regulate the molecular events in cancer. We also reviewed the current knowledge and recent finding regarding the association of circRNA with head and neck cancer. We briefly discussed the potential of circRNA as diagnostic biomarkers as well as the possibility for therapeutic targeting.
\end{abstract}

\section{Commentary}

With rapid advances in molecular biology and unrevealing of human genome and transcriptome, new regulatory roles of noncoding RNA molecules have been identified, and complexity of gene expression dynamics in cancer pathology has been shown. circularRNAs (cirRNAs) are highly conserved and stable covalently closed RNA circles with gene-regulatory potential [1,2]. Although circular RNA was first described a while ago, until recently they were considered as a byproduct of splicing process with no biological importance. Serval regulatory roles in cancer have been attributed to circRNA [1-3]. Biogenies and function of cirRNAs are depicted in Figure 1. circRNAs can act as miRNA sponges or decoys. miRNA-Ago complex can bind to the cirRNAs, and this interaction may lead to liberating the target mRNAs from miRNA-Ago complex which leads to a more efficient translation. circRNAs can also act as RNA binding protein (RBP) sponge or decoy. Some of circRNAs have binding motifs for both enzyme and their substrates and can enable the kinetics. circRNAs that originate from exon-intron junctions can enhance the transcription of neighboring genes through interaction with U1 snRNP. Splicing and

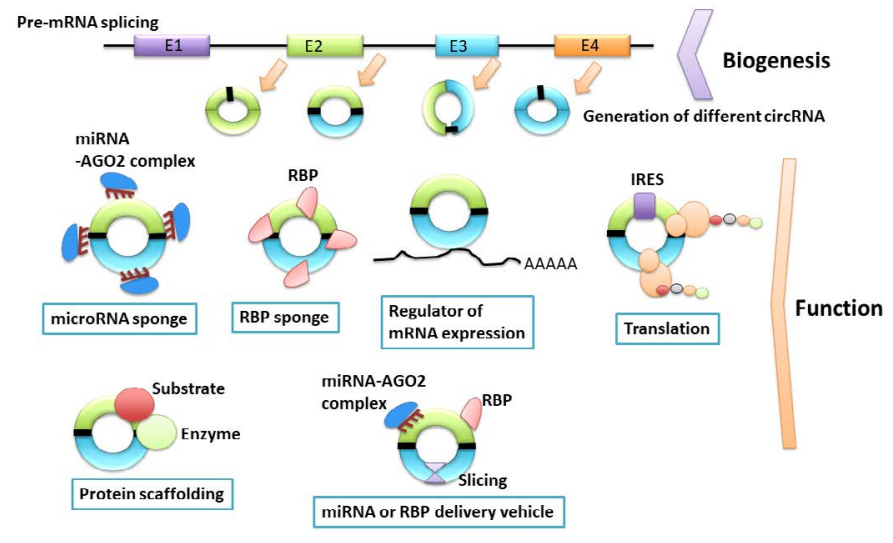

Figure 1. Biogenesis and functions of circular RNA transcription of many genes may also be indirectly regulated through a competition between canonical splicing and back splicing. Some circRNAs have been shown to regulate translation. circRNAs can be genetically modified as delivery vehicle for miRNA-Ago complex or RBP [1-3].

It has been evidenced that circRNAs play pivotal roles in regulating proliferation, angiogenesis, evading cellular senescence, and metastasis in cancer $[3,4]$. Most circRNAs exhibit tissue-specific properties and are stable in biofluids [5]. circRNAs are resistant to RNases and can be detected by probes and quantitative PCRs [1]. These characteristics enable them as promising biomarkers for diagnosis and monitoring of different cancers including head and neck cancer. The stability and capacity of circRNA to sponge proteins and mRNAs make them attractive therapeutic vehicles for therapeutic purposes. For instance, specific miRNA and protein binding sites can be specially designed and targeted by circRNA to modulate oncogenic profile.

Recent investigate indicated the dysregulation of circRNA in various forms of head and neck cancer. circRNA_100290 was shown to be overexpressed in oral squamous cell carcinoma compared to the controls and function as a sponge for the miRNA-29 family which leads to the regulation of cell division protein kinase 6 (CDK6) which is one of the master regulators of cancer-induced cell cycle changes [6]. In laryngeal cancer, a significant increase in hsa_circRNA_100855 level is reported in the tumor tissue compared to the healthy tissue [7]. Patients with T3 and T4 stage, neck lymph node metastasis or

${ }^{*}$ Correspondence to: Fatemeh Momen-Heravi, Section of Oral, Diagnostic, and Rehabilitation Sciences, Columbia University, College of Dental Medicine, 630 W. 168 St., New York, NY 10032, USA, Tel 00-1-508-856-6794, Fax: 00-1-508856-4770, E-mail: shashi.bala@umassmed.edu

Key words: circRNA function, diagnostic biomarkers, hallmark cance, gene expression

Received: June 11, 2018; Accepted: June 25, 2018; Published: June 29, 2018 
advanced clinical stage showed higher levels of hsa_circRNA_100855 expression [7]l. The hsa_circRNA_104912 level was significantly lower in laryngeal squamous cell carcinoma than in corresponding adjacent non-neoplastic tissues [7]. Patients with T3-4 stage, neck nodal metastasis, poor differentiation or advanced clinical stage had a lower hsa_circRNA_104912 expression [7].

In another study, Verduci et al. [8], using a sample consisted of 115 head and neck squamous cell carcinoma patients, reported that circPVT1 is over-expressed in tumors compared to matched nontumoral tissues [8]. The overexpression was mainly related to TP53 mutation status and was related to a more malignant phenotype. Mechanistically, the circPVT1 expression is transcriptionally increased by the mut-p53/YAP/TEAD complex [8]. circPVT1 modulated the gene involved in cell proliferation [8]. Xia et al. [9] reported overexpression of hsa_circ_0067934 in esophageal cancer cells compared to the adjacent noncancerous tissue samples. The expression levels were related to poor tumor differentiation and late tumor staging and higher involvement of lymph nodes [9].

In conclusion, emerging evidence indicates that circRNAs function as important drivers of cancer. However, this line of research is still emerging. Future studies should focus on mechanistic characterization and causal association of circRNAs in tumorigenesis in head and neck cancer. Parallel with mechanistic studies, well-controlled clinical studies are needed to assess the utility of circRNAs for diagnosis and therapeutic purposes. Based on the recent reports, circRNAs can be as potentially promising candidates not only as a biomarker for cancer diagnosis but also a potential monitoring and therapeutic targets.

Various circular RNAs (circRNAs) can be generated from a same genomic locus. circRNAs are formed by non-canonical splicing process. circRNAs consist of one or more exons and can also have unspliced intronic sequences. Colored bars (exons) black lines (introns). In recent years various roles of circRNAs in cellular processes is arising such as miRNA sponge, RBP sponge, regulator of mRNA expression and translation, protein scaffolding and as delivery vehicles for miRNA-Ago complex or RBP proteins.

\section{Author contributions}

FMH and SB both contributed equally to drafting the work. Both authors contributed to concept, writing, and final approval.

\section{References}

1. Kristensen LS, Hansen TB, Venø MT, Kjems J (2018) Circular RNAs in cancer opportunities and challenges in the field. Oncogene 37: 555-565. [Crossref]

2. Chen LL (2016) The biogenesis and emerging roles of circular RNAs. Nat Rev Mol Cell Biol 17: 205-211. [Crossref]

3. Momen-Heravi F, Bala S (2018) Emerging role of non-coding RNA in oral cancer. Cell Signal 42: 134-143. [Crossref]

4. Qu S, Liu Z, Yang X, Zhou J, Yu H, et al. (2018) The emerging functions and roles of circular RNAs in cancer. Cancer Lett 414: 301-309. [Crossref]

5. Xu Z, Yan Y, Zeng S, Dai S, Chen X, et al. (2017) Circular RNAs: clinical relevance in cancer. Oncotarget 9: 1444-1460. [Crossref]

6. Chen L, Zhang S, Wu J, Cui J, Zhong L, et al. (2017) circRNA_100290 plays a role in oral cancer by functioning as a sponge of the miR-29 family. Oncogene 36: 4551-4561. [Crossref]

7. Xuan L, Qu L, Zhou H, Wang P, Yu H, et al. (2016) Circular RNA: a novel biomarker for progressive laryngeal cancer. Am J Transl Res 8: 932-939. [Crossref]

8. Verduci L, Ferraiuolo M, Sacconi A, Ganci F, Vitale J, et al. (2017) The oncogenic role of circPVT1 in head and neck squamous cell carcinoma is mediated through the mutant p53/YAP/TEAD transcription-competent complex. Genome Biol 18: 237. [Crossref]

9. Xia W, Qiu M, Chen R, Leng X, Wang J, et al. (2016) Circular RNA has circ 0067934 is upregulated in esophageal squamous cell carcinoma and promoted proliferation. $\mathrm{Sci}$ Rep 6: 35576. [Crossref]

Copyright: (C2018 Momen-Heravi F. This is an open-access article distributed under the terms of the Creative Commons Attribution License, which permits unrestricted use, distribution, and reproduction in any medium, provided the original author and source are credited. 